\title{
Optimal Transportation Flows
}

\author{
R. Klötzler
}

\begin{abstract}
This paper deals with a modification of L. C. Young's flow concept in application to transportation flow problems. There will be proved necessary and sufficient conditions for an optimal transport and its dual deposit problem.
\end{abstract}

Keywords: Transportation flow problems, deposit problems, dual optimization problems, generalized Pontryagin's maximum principle

AMS subject classiflcation: $49 \mathrm{~N} 15,49 \mathrm{Q} 15,49 \mathrm{Q} 20$

\section{Introduction}

In 1969 L. C. Young [17] introduced a fundamental new concept in the calculus of variations which is based on substitution of curves and surfaces by so-called "generalized curves" and "generalized flows". He represents these generalized objects either by special subclasses of regular Borel measures or by $C^{*}(A)$, the dual of $C(A)$, the space of continuous functions on a fixed bounded closed Euclidean set $A$.

Here, we prefer an alternative approach highly based on the ideas of L. V. Kantorovič, M. K. Gawurin and G. S. Rubinstein (see [7] and [8]) on freight flow analysis and the use of proper set functions of bounded variation from $L_{\infty}^{n m}(\Omega)^{*}$, where $\Omega$ is a bounded strongly Lipschitz domain of $\mathbb{E}^{m}$ and $n$ denotes the number of different transport products within $\Omega$. However, contrary to this mentioned Russian model we do not assume the knowledge of the global cost of transport between two points $A$ and $B$ within $\Omega$, but only the local cost rate (dependent on the place and the direction of transport), which seems to be more realistic. First publications of this concept by the author are referenced in [11 - 13].

Let $\mathfrak{B}$ be the $\sigma$-algebra of all Lebesgue-measurable subsets of $\Omega$ and $\alpha_{k}(k=$ $1, \ldots, n$ ) finite Borel measures on $\mathfrak{B}$ which are equilized by the assumption

$$
\int_{\Omega} d \alpha_{k}=0 \quad(k=1, \ldots, n) .
$$

We interprete $\alpha_{k}(e)$ for each $e \in \mathfrak{B}$, positive or negative, as the given consumption or production rate of $e$, respectively, with respect to the $k$-th product. We understand this system as a transport realized by a stationary (= independently of time) vector flow within $\Omega$. We describe it by a vectorial set function $\mu=\left(\mu_{1}, \ldots, \mu_{n}\right)$, where

R. Klötzler: Univ. Leipzig, Institut für Mathematik, Augustusplatz 10, D - 04109 Leipzig 
$\mu_{k}(e) \in \mathbb{E}^{m}$ expresses the vector of average flow of the $k$-th product on the set $e$ dimensioned as transported mass times (average) velocity. We make the reasonable assumptions $\mu_{k} \in L_{\infty}^{m}(\Omega)^{*}$ or $\mu \in L_{\infty}^{n m}(\Omega)^{*}$, respectively, such that $\mu_{k}$ represents an additive set function on $\mathfrak{B}$ of bounded variation with the property that $\mu_{k}(e)=0$ if the Lebesgue measure meas $e$ is zero (comp. $[5,6]$ ). Then, by a well-known representation theorem (see [5: p. 258] and [6: p. 194]), each linear continuous functional on $L_{\infty}^{m}(\Omega)^{*}$ can be represented via Radon integrals by

$$
\left\langle u^{k}, \mu_{k}\right\rangle=\int_{\Omega} u^{k}(x)^{\top} d \mu_{k}(x) \quad \text { for all } u^{k} \in L_{\infty}^{m}(\Omega)
$$

with a uniquely determined $\mu_{k} \in L_{\infty}^{m}(\Omega)^{*}$ (up of equivalence). Here and later, the norm in $L_{\infty}^{m}(\Omega)$ is given by

$$
\left\|u^{k}\right\|_{L_{\infty}^{m}(\Omega)}=\sqrt{\sum_{j=1}^{m}\left\|u_{j}^{k}\right\|_{L_{\infty}(\Omega)}^{2}}
$$

for

$$
u^{k}=\left(u_{1}^{k}, \ldots, u_{m}^{k}\right) \quad \text { with } u_{j}^{k} \in L_{\infty}(\Omega) \quad(k=1, \ldots, n ; j=1, \ldots, m)
$$

Then, each linear continuous functional on $L_{\infty}^{n m}(\Omega)$ has the form

$$
\langle u, \mu\rangle=\int_{\Omega} u^{\top}(x) d \mu(x)=\int_{\Omega} \sum_{k=1}^{n} u^{k}(x)^{\top} d \mu_{k}(x)
$$

for any

$$
u=\left(u^{1}, \ldots, u^{n}\right) \quad \text { with } u^{k} \in L_{\infty}^{m}(\Omega)(k=1, \ldots, n) .
$$

We say that $\mu \in L_{\infty}^{n m}(\Omega)^{*}$ is a feasible flow (with respect to $\alpha=\left(\alpha_{1}, \ldots, \alpha_{n}\right)$ ) provided it satisfies the variational equalities (for $k=1, \ldots, n$ )

$$
\int_{\Omega} \nabla \sigma^{\top} d \mu_{k}(x)=\int_{\Omega} \sigma(x) d \alpha_{k}(x) \quad \text { for all } \sigma \in W_{\infty}^{1}(\Omega)
$$

which we can interprete as the "continuity equation" for the $k$-th product under consideration of the given $\alpha_{k}$ (cp. [11]). Namely, if $\alpha_{k}$ possesses a summable density $\varrho_{k}$ and $\mu_{k}$ a continuously differentiable density $y_{k}$, then we obtain from $(5), d \alpha_{k}(x)=\varrho_{k}(x) d x$ and $d \mu_{k}(x)=y_{k}(x) d x$ the equivalent requirements

$$
\operatorname{div} y_{k}(x)=-\varrho_{k}(x) \quad \text { in } \Omega \quad \text { and }\left.\quad y_{k}^{\top}(x) n(x)\right|_{\partial \Omega}=0 .
$$

whereby $n(x)$ denotes the exterior unit normal vector of $\partial \Omega$ in $x$. We denote the linear affine subset of all feasible flows $\mu$ by $Y$ and by $Y_{0}$ the subspace of all $\mu \in L_{\infty}^{m n}(\Omega)^{*}$ satisfying the corresponding homogeneous form of (5). 
Finally, we evaluate each feasible flow $\mu$ by means of a given local cost rate $r$ on $\Omega \times \mathbb{E}^{n m}$ with the following basic properties:

$\left.\begin{array}{l}\text { - } r(\cdot, v) \text { is summable on } \Omega \\ \text { - } r(x, \cdot) \text { is positiv homogeneous of degree one and convex on } \mathbb{E}^{n m} \forall x \in \Omega \\ \text { - } \gamma_{1}|v| \leq r(x, v) \leq \gamma_{2}|v|\left(v \in \mathbb{E}^{m n}, x \in \Omega\right) \text { for some constants } \gamma_{1}, \gamma_{2}>0 \text {. }\end{array}\right\}$

We do this by using the cost functional

$$
\int_{\Omega} r(x, d \mu(x))=\sup \left\{\langle u, \mu\rangle \mid u \in L_{\infty}^{n m}(\Omega), u^{\top}(x) v \leq r(x, v) \forall v \in \mathbb{E}^{n m}\right\} .
$$

We introduce the denotation

$$
\mathfrak{F}(x)=\left\{z \in \mathbb{E}^{n m} \mid z^{\top} v \leq r(x, v) \text { for all } v \in \mathbb{E}^{n m}\right\}
$$

called, in conformity with C. Carathéodory [2: p. 101], the figuratrix set with respect to $r$. By using F. H. Clarke's generalized gradient of $r$ with respect to $v[4: p .27$ ] that implies that $\mathfrak{F}(x)=\partial_{v} r(x, 0)$. So, $r(x, \cdot)$ is said to be the support function of $\mathfrak{F}(x)$.

The generalized integral definition (7) seems to be reasonable from the point of view that for given $\mu \in L_{1}^{n m}(\Omega) \subset L_{\infty}^{n m}(\Omega)^{*}$ there is a unique summable density $y$ with $d \mu(x)=y(x) d x$ and a function $u_{*} \in L_{\infty}^{n m}(\Omega)$ with $u_{*}^{\top}(x) y(x)=r(x, y(x))$ and $u_{*}(x) \epsilon$ $\mathfrak{F}(x)$ such that $\int_{\Omega} r(x, d \mu(x))$ coincides with the Lebesgue integral $\int_{\Omega} r(x, y(x)) d x$. We summarize this in the following Lemma.

Lemma 1: For all absolutely continuous flows $\mu \in Y$ there is a unique corresponding density $y \in L_{1}^{n m}(\Omega)$ such that

$$
\int_{\Omega} r(x, d \mu(x))=\int_{\Omega} r(x, y(x)) d x
$$

holds. If we denote the set of all those $y$ by $Y^{\prime}$, then

$$
\inf _{\mu \in Y} \int_{\mathbf{\Omega}} r(x, d \mu(x)) \leq \inf _{y \in Y^{\prime}} \int_{\Omega} r(x, y(x)) d x .
$$
by

Now, following. [11 - 13], we can formulate our transportation flow problem (TFP)

$$
K(\mu):=\int_{\Omega} r(x, d \mu(x)) \longrightarrow \min \text { on } Y .
$$

It is easily seen that this is a convex optimization problem. 


\section{The dual deposit problem}

For each $\mu \in Y$, we get from the definition (7) immediately

$$
\int_{\Omega} r(x, d \mu(x)) \geq\langle\nabla S, \mu\rangle
$$

if the vector-valued function $S=\left(S^{1}, \ldots, S^{n}\right)$ belongs to $W_{\infty}^{1, n}(\Omega)$ and satisfies the constraint

$$
\nabla S(x) \in \mathfrak{F}(x) \quad \text { for a.e. } x \in \Omega \text {. }
$$

Therefore, with $\sigma=S^{k}$, we conclude from (5), (9) and (10) that

$$
K(\mu) \geq \int_{\Omega} \sum_{k=1}^{n} S^{k}(x) d \alpha_{k}(x)=\int_{\Omega} S^{\top}(x) d \alpha(x)=: K_{D}(S) .
$$

This inequality characterizes the optimization problem

$$
K_{D}(S)=\int_{\Omega} S^{\top}(x) d \alpha(x) \longrightarrow \max \text { on } W_{\infty}^{1, n}(\Omega)
$$

under the constraint (11) as a dual problem of the (TFP)-problem (9) in the sense of [2: p. 341].

We can interprete (13) as a "deposit problem" for constructing $n$ hills $z=S^{k}(x)$ on $\Omega(k=1, \cdots, n)$ under slope conditions (11) such that for given store prices $\alpha_{k}$ the store profit $K_{D}(S)$ is maximal. In specialized form the duality between (9) and (13) is one of the oldest examples of dual optimization problems and was already discovered by G. Monge [14] and P. Appell [1].

Problem (13) is a usual multidimensional problem of optimal control of the Dieudonné -Rashevsky type according to L. Cesari [3]. By (1), without loss of generality we can reduce the study of problem (13) to the standardized set

$$
\mathfrak{S}=\left\{S \in W_{\infty}^{1, n}(\Omega) \mid \nabla S(x) \in \mathfrak{F}(x) \text { for a.e. } x \in \Omega, S\left(\hat{x}_{0}\right)=0\right\}
$$

where $\hat{x}_{0}$ is any given point in $\Omega$.

Theorem 1: The deposit problem (13) has an optimal solution.

Proof: As a consequence of the basic assumptions (6) on $r$ the figuratrix sets $\mathfrak{F}(x)$ are uniformly bounded in $\mathbb{E}^{n m}$ with respect to $x \in \Omega$. They are closed and convex. Moreover, the origin $\mathbf{0}$ of $\mathbb{E}^{n m}$ is an interior point of each $\mathfrak{F}(x)$. Therefore, by Sobolev's imbedding theorems, $\mathfrak{S}$ is a closed and weakly compact subset in $W_{p}^{1, n}(\Omega)$ for each $p>m$. The same reasoning implies that $K_{D}(S)$ is a linear continuous functional on $W_{p}^{1, n}(\Omega)$. By the generalized existence theorem of Weierstrass, this guarantees the existence of an optimal solution $S_{0}$ of problem (13) at first in $W_{p}^{1, n}(\Omega)$. Since $S_{0}$ satisfies (11), it belongs also to $W_{\infty}^{1, n}(\Omega)$ 
Now, we denote again an optimal solution of problem (13) by $S_{0}$. First, we can apply to $S_{0}$ a generalized $\varepsilon$-Pontryagin maximum principle due to R. Klötzler [9] and $S$. Pickenhain [10]. With reference to these papers the following necessary optimality condition holds.

Theorem 2: If $S_{0}$ is an optimal solution of problem (13), then for each $\varepsilon>0$.there is a vector $y \in L_{2}^{n m}(\Omega)$ (depending on $\varepsilon$ ) with

$$
\varepsilon+\int_{\Omega} y^{\top}(x)\left(\nabla S_{0}(x)-u(x)\right) . d x \geq 0 \quad \text { for all } u \in \dot{L}_{\infty}^{m n}(\Omega),
$$

$u(x) \in \mathfrak{F}(x)$ a.e. on $\Omega$, and

$$
\int_{\Omega} \nabla \sigma^{\top}(x) y(x) d x=\int_{\Omega} \sigma(x)^{\top} d \alpha(x) \quad \text { for all } \sigma \in W_{\infty}^{1, n}(\Omega) .
$$

Now, we replace the number $\varepsilon$ by $1 / \kappa(\kappa \in \mathbb{N})$ in Theorem 2 and denote the corresponding $y$ by $y_{\kappa}$. For this sequence $\left\{y_{\kappa}\right\}$ we obtain from (15) for any feasible $u$ and $\kappa \in \mathbb{N}$ the inequality

$$
\frac{1}{\kappa}+\int_{\Omega} \nabla S_{0}^{\top}(x) y_{\kappa}(x) d x \geq \int_{\Omega} u(x)^{\top} y_{\kappa}(x) d x .
$$

Because of the basic assumptions on $r$ and (16), especially for $\sigma=S_{0}$, inequality (17) leads to

$$
\begin{aligned}
& \frac{1}{\kappa}+\int_{\Omega} S_{0}^{\top}(x) d \alpha(x) \\
& \quad \geq \sup \left\{\int_{\Omega} u(x)^{\top} y_{\kappa}(x) d x\left|u \in L_{\infty}^{n m}(\Omega),\right| u(x) \mid=\gamma_{1} \text { a.e. on } \Omega\right\} \\
& \quad=\gamma_{1} \int_{\Omega}\left|y_{\kappa}(x)\right| d x
\end{aligned}
$$

This shows the boundedness of the sequence $\left\{y_{\kappa}\right\}$ in $L_{1}^{n m}(\Omega)$ such that the theorem of Alaoglu (see [15: p. 150]) implies the existence of a weak ${ }^{*}$-convergent subsequence $\left\{y_{\kappa^{\prime}}\right\}$ in $L_{1}^{n m}(\Omega) \subset L_{\infty}^{n m}(\Omega)^{*}$ with $y_{\kappa^{\prime}} \rightarrow^{*} \mu_{0} \in L_{\infty}^{n m}(\Omega)^{*}$ for $\kappa^{\prime} \rightarrow \infty$. Hence, taking the limit $\kappa^{\prime} \rightarrow \infty$ in (16) and (17), we conclude

$$
\int_{\Omega} \nabla S_{0}^{\top}(x) d \mu_{0}(x) \geq \int_{\Omega} u(x)^{\top} d \mu_{0}(x) \quad \text { fọr all } u \in, L_{\infty}^{n m}(\Omega)
$$

with the constraint $u(x) \in \mathfrak{F}(x)$ a.e. on $\Omega$, as well as

$$
\int_{\Omega} \nabla \sigma^{\top}(x) d \mu_{0}(x)=\int_{\Omega} \sigma(x)^{\top} d \alpha(x) \quad \text { for all } \sigma \in W_{\infty}^{1, n}(\Omega) .
$$

In total we have proved the following maximum principle. 
Theorem 3: If $S_{0}$ is an optimal solution of the deposit problem (13), then there is a set function $\mu_{0} \in L_{\infty}^{n m}(\Omega)^{*}$ which satisfies the conditions (19) and (20).

Obviously, in case $\mu=\left(\mu_{1}, \ldots, \mu_{n}\right)=\mu_{0}$ condition (20) is equivalent to the system (5). This has the consequence that $\mu_{0} \in Y$. The formulas (19) and (20) together, applied for $\sigma=S_{0}$, yield by (7) and (12) the result $K\left(\mu_{0}\right)=K_{D}\left(S_{0}\right)$ and allow the following conclusion.

Theorem 4: Between the transportation flow problem (9) and the corresponding deposit problem (13) strong duality is guaranted in the sense of $\min _{Y} K(\mu)=$ $\max _{\mathfrak{S}} K_{D}(S)$.

\section{Special consequences of optimality}

In this section we intend to get more information about the structure of the optimal solutions $\mu_{0}$ for the problem (TFP) and $S_{0}$ for the deposit problem, respectively.

Theorem 5: If $r$ is continuous and $\mu_{0} \in Y$ has a summable density $y_{0}$ in a neighbourhood $B\left(x^{*}, \delta\right) \subset \Omega$ of $x^{*}$, then for almost all $x \in B\left(x^{*}, \delta\right)$ the pointwise maximum principle

$$
\nabla S_{0}^{\top}(x) y_{0}(x) \geq v^{\top} y_{0}(x) \quad \text { for all } v \in \mathfrak{F}(x)
$$

holds.

Proof: We consider an arbitrary point $x^{0} \in B\left(x^{*}, \delta\right)$ where $\nabla S_{0}\left(x^{0}\right)$ exists and which is a Lebesgue point of $y_{0}$ and $\nabla S_{0}$. We choose an arbitrary $v \in \mathfrak{F}\left(x^{0}\right)$ and construct a continuous function $\tilde{u} \in L_{\infty}^{m n}\left(B\left(x^{0}, \varrho\right)\right)$ with $B\left(x^{0}, \varrho\right) \subset B\left(x^{*}, \delta\right), \tilde{u}(x) \in \mathfrak{F}(x)$ and $\tilde{u}\left(x^{0}\right)=v$. The continuity of $r$ guarantees the existence of such an $\tilde{u}$. According to the assumptions of Theorem 5 we get from (19) for

$$
u(x)= \begin{cases}\tilde{u}(x) & \text { in } B\left(x^{0}, \varrho\right) \\ \nabla S_{0}(x) & \text { elsewhere in } \Omega\end{cases}
$$

the inequality

$$
\int_{B\left(x^{0}, \varrho\right)} \nabla S_{0}(x)^{\top} y_{0}(x) d x \geq \int_{B\left(x^{0}, \varrho\right)} u(x)^{\top} y_{0}(x) d x .
$$

Dividing this inequality by meas $B\left(x^{0}, \varrho\right)$ and taking the limit $\varrho \rightarrow 0$, we get

$$
\nabla S_{0}^{\top}\left(x^{0}\right) y_{0}\left(x^{0}\right) \geq v^{\top} y_{0}\left(x^{0}\right) \quad \text { for all } v \in \mathfrak{F}\left(x^{0}\right) .
$$

Since $x^{0}$ is arbitrary choosen in $B\left(x^{*}, \delta\right)$ this result expresses the validity of (21)

Remark 1: Because of (21) and the definition of the figuratrix set $\mathfrak{F}(x)$ in connection with (8) and (14) we deduce

$$
\nabla S_{0}(x)^{\top} y_{0}(x)=\max _{v \in \mathcal{F}(x)} v^{\top} y_{0}(x)=r\left(x, y_{0}(x)\right)
$$


as well as

$$
\nabla S_{0}(x)^{\top} v \leq r(x, v) \quad \text { for all } v \in \mathbb{E}^{n m} .
$$

Both together implies the inequality

$$
r(x, v)-r\left(x, y_{0}(x)\right) \geq \nabla S_{0}^{\top}(x)\left(v-y_{0}(x)\right) \quad \text { for all } v \in \mathbb{E}^{n m} .
$$

This gives, in accordance with (21), the inclusion

$$
\nabla S_{0}(x) \in \partial_{v} r\left(x, y_{0}(x)\right) \text {. }
$$

The inclusion (23) allows in a further special case the following important conclusion.

Theorem 6: We assume $r$ to be continuous and $r(x, v)$ to be differentiable with respect to $v \neq 0$. If the optimal solution $\mu_{0}$ of the (TFP) has a summable density $y_{0}$ in $\Omega$, then there exists a vector function $S_{0} \in \mathfrak{S}$ with the property (23) a.e. on $\Omega$. In points $x \in \Omega$, where $y_{0}(x) \neq 0$ holds, the condition

$$
\nabla S_{0}(x)=r_{v}\left(x, y_{0}(x)\right)
$$

is valid a.e.

Remark 2: Obviously, condition (24) holds also on a subdomain $\Omega_{0} \subset \Omega$ if $\mu_{0}$ has a summable density $y_{0}(x) \neq \mathbf{0}$ on $\Omega_{0}$.

Now we continue our investigation under the assumption of Remark 2 and suppose that $r$ and $r_{v}$ are continuously differentiable outside of $v=0$. Additionally, we require $y_{0} \in C^{1}\left(\Omega_{0}\right)$. Then, (24) implies that $\nabla S_{0} \in C^{1}\left(\Omega_{0}\right)$. Moreover, the positive homogeneity of $r(x, \cdot)$ gives

$$
r\left(x, y_{0}(x)\right)=\nabla S_{0}(x)^{\top} y_{0}(x) \quad \text { on } \Omega_{0} .
$$

We differentiate both sides of (25) with respect to $x^{k}$ and obtain

$$
\begin{aligned}
r_{x^{k}}\left(x, y_{0}(x)\right) & +\sum_{\beta=1}^{n} \sum_{i=1}^{m} r_{v_{\beta}^{i}}\left(x, y_{0}(x)\right) y_{0 \beta x^{k}}^{i}(x) \\
& =\sum_{\beta=1}^{n} \sum_{i=1}^{m} S_{0 x^{i} x^{k}}^{\beta}(x) y_{0 \beta}^{i}(x)+\sum_{\beta=1}^{n} \sum_{i=1}^{m} S_{0 x^{i}}^{\beta}(x) y_{0 \beta x^{k}}^{i}(x)
\end{aligned}
$$

and consequently, using (24),

$$
r_{x^{k}}\left(x, y_{0}(x)\right)=\sum_{\beta=1}^{n} \sum_{i=1}^{m} \frac{\partial r_{v_{\beta}^{k}}\left(x, y_{0}(x)\right)}{\partial x^{i}} y_{0 \beta}^{i}(x) \quad \text { for } \quad k=1, \ldots, m
$$

We shall refer to these equations (26) as the system of "Euler's flow equations". In summary we obtain the following assertion. 
Theorem 7: Let $\mu_{0}$ be an optimal solution of the (TFP), $r \in C^{1}$ and $r_{v} \in C^{1}$, and suppose the existence of a continuously differentiable density $y_{0}$ of $\mu_{0}$ with the property $y_{0}(x) \neq 0$ in the subdomain $\Omega_{0}$. Then, $y_{0}$ has to satisfy Euler's flow equations (26) in $\Omega_{0}$.

Another consequence of (24) is the following result.

Theorem 8: Let $\mu_{0}$ be an optimal solution of the (TFP), $r \in C^{1}$ and $r_{v} \in C^{1}$, and suppose the existence of a piecewise continuous density $y_{0}$ of $\mu_{0}$ in $\Omega_{0}$ where $y_{0}(x) \neq 0$ on $\Omega_{0}$ is valid. We assume that the jumps of yo are only at a smooth hypersurface $\Gamma$ which divides $\Omega_{0}$ into two parts $\Omega_{0}^{\prime}$ and $\Omega_{0}^{\prime \prime}$. Then, in each point $x \in \Gamma$ for every tangent vector $\mathrm{t}$ of $\Gamma$

$$
\left[r_{v_{\beta}}\left(x, y_{0}^{\prime}(x)\right)-r_{v_{\beta}}\left(x, y_{0}^{\prime \prime}(x)\right)\right]^{\top} \mathfrak{t}=0 \quad(\beta=1, \ldots, n)
$$

holds, whereby $y_{0}^{\prime}$ and $y_{0}^{\prime \prime}$ are the limits

$$
y_{0}^{\prime}(x)=\lim _{\Omega_{0}^{\prime} \ni x_{k}^{\prime} \rightarrow x} y_{0}\left(x_{k}^{\prime}\right) \quad \text { and } \quad y_{0}^{\prime \prime}(x)=\lim _{\Omega_{0}^{\prime \prime} \ni x_{k}^{\prime \prime} \rightarrow x} y_{0}\left(x_{k}^{\prime \prime}\right) .
$$

Proof: Let $\mathfrak{B}$ be a smooth curve on $\Gamma$ starting from $x_{0} \in \Gamma$ with the initial direction $\mathfrak{t}$ such that for its analytical representation $x=\psi(\tau)$ there hold $x_{0}=\psi(0)$ and $\dot{\psi}(0)=\mathfrak{t}$. Since $S_{0}^{\beta}$ is continuous, we get from (24)

$$
\begin{aligned}
S_{0}^{\beta}(\psi(\tau))-S_{0}^{\beta}\left(x_{0}\right) & =\int_{0}^{\tau} \nabla S^{\beta}(\psi) \dot{\psi}(\tau) d \tau \\
& =\int_{0}^{\tau} r_{v_{\beta}}\left(\psi, y_{0}^{\prime}(\psi)\right)^{\top} \dot{\psi}(\bar{\tau}) d \bar{\tau} \\
& =\int_{0}^{\tau} r_{v_{\beta}}\left(\psi, y_{0}^{\prime \prime}(\psi)\right)^{\top} \dot{\psi}(\bar{\tau}) d \bar{\tau}
\end{aligned}
$$

and, via differentiation by $\tau$ at $\tau=0,\left[r_{v_{\rho}}\left(x_{0}, y_{0}^{\prime}\left(x_{0}\right)\right)-r_{v_{\rho}}\left(x_{0}, y_{0}^{\prime \prime}\left(x_{0}\right)\right)\right] \mathfrak{t}=0$

\section{An example}

It goes without saying that in general one can not expect to find elementar solutions of the (TFP) by analytic expressions. For such cases we propose approximate procedures by finite element methods according to the concepts of [11 - 13]. Nevertheless, for an illustration of our theory we shall consider at least one example.

Let be given the rectangle $\Omega=[0, a] \times[0, b]$ in $\mathbb{E}^{2}$, two homogeneous sources $\overline{O Q_{1}}$ and $\overline{Q_{2} Q_{3}}$ for $x^{1}=0$ of different products and directly across two homogeneous sinks $\overline{Z_{0} Z_{1}}$ and $\overline{Z_{2} Z_{3}}$ for $x^{1}=a$ (see Fig. 1). We put

$$
r(x, v)=\sqrt{\left|v_{1}\right|^{2}+\left|v_{2}\right|^{2}} \quad \text { for } \quad v_{i} \in \mathbb{E}^{2} \quad(i=1,2)
$$


and characterize $\alpha_{1}$ and $\alpha_{2}$ by the variational equations

$$
\begin{aligned}
& \int_{\Omega} \sigma(x) d \alpha_{1}(x)=\int_{0}^{b_{1}}\left(\sigma\left(a, x^{2}\right)-\sigma\left(0, x^{2}\right)\right) d x^{2} \\
& \int_{\Omega} \sigma(x) d \alpha_{2}(x)=\int_{b_{2}}^{b}\left(\sigma\left(a, x^{2}\right)-\sigma\left(0, x^{2}\right)\right) d x^{2}
\end{aligned}
$$

Furthermore, we require

$$
b_{2}-b_{1} \geq 2 a
$$

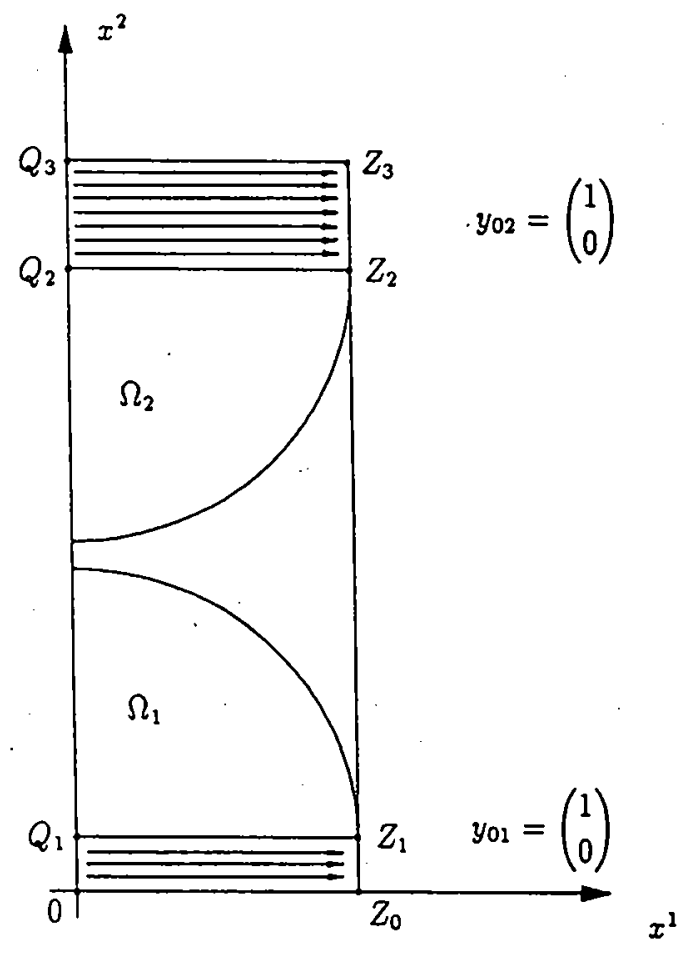

$$
\begin{gathered}
Q_{1}=\left(0, b_{1}\right), \quad Q_{2}=\left(0, b_{2}\right), \quad Q_{3}=(0, b) \\
Z_{0}=(a, 0), \quad Z_{1}=\left(a, b_{1}\right), \quad Z_{2}=\left(a, b_{2}\right), \quad Z_{3}=(a, b)
\end{gathered}
$$

Figure 1

In this special case assumption (1) is satisfied, the constraint (11) means

$$
\left|\nabla S^{1}\right|^{2}+\left|\nabla S^{2}\right|^{2} \leq 1
$$


the condition (24) says

$$
S_{0 x^{k}}^{\beta}(x)=\frac{y_{0 \beta}^{k}(x)}{\sqrt{\left|y_{01}(x)\right|^{2}+\left|y_{02}(x)\right|^{2}}} \quad(\beta, k=1,2)
$$

if $\left|y_{01}(x)\right|^{2}+\left|y_{02}(x)\right|^{2}>0$, and Euler's flow equations defined as in (26) are given by

$$
0=\sum_{i, \beta=1}^{2}\left(\frac{y_{0 \beta}^{k}}{\sqrt{\left|y_{01}\right|^{2}+\left|y_{02}\right|^{2}}}\right)_{x^{i}} y_{0 \beta}^{i} \quad \text { for } \quad k=1,2 .
$$

We confirm very easily the following solution of (TFP) (cp. Figure 1):

$$
\begin{aligned}
& y_{01}(x)= \begin{cases}\left(\begin{array}{l}
1 \\
0
\end{array}\right) & \text { for } x^{2}<b_{2} \\
\mathbf{0} & \text { elsewhere }\end{cases} \\
& y_{02}(x)= \begin{cases}\left(\begin{array}{l}
1 \\
0
\end{array}\right) & \text { for } x^{2} \geq b_{2} \\
\mathbf{0} & \text { elsewhere }\end{cases} \\
& S_{0}^{1}(x)= \begin{cases}x^{1} & \text { for } x^{2} \leq b_{1} \\
\sqrt{\left(x^{1}\right)^{2}+\left(x^{2}-b_{1}\right)^{2}} & \text { in the sector } \Omega_{1} \\
a & \text { elsewhere }\end{cases} \\
& S_{0}^{2}(x)= \begin{cases}x^{1} & \text { for } x^{2} \geq b_{2} \\
\sqrt{\left(x^{1}\right)^{2}+\left(x^{2}-b_{2}\right)^{2}} & \text { in the sector } \Omega_{2} \\
a & \text { elsewhere. }\end{cases}
\end{aligned}
$$

One proves immediately, by using Lemma 1 and the notation $\mu_{0}(e)=\int_{e} y_{0}(x) d x \quad(e \in$ $\mathfrak{B})$, the equation

$$
K\left(\mu_{0}\right)=\left(b-b_{2}\right) a+b_{1} a=K_{D}\left(S_{0}\right) .
$$

This guarantees in virtue of Theorem 1 the optimality of $\mu_{0}$ and $S_{0}$ with respect to our (TFP) and its dual problem, respectively. .

Remark 3: For the construction of the given solution, the requirement (28) is essential. If we have a smaller distance $b_{2}-b_{1}<2 a$, then the optimal flows $y_{01}$ and $y_{02}$ depend on attractions, which will be discussed in a next separate publication.

\section{References}

[1] Appell, P.: Le problème géomètrique de deblais et remblais (Memorial des Sciences Mathématiques: Vol. 27). Paris: Gauthier-Villars 1928.

[2] Carathéodory, C.: Variationsrechnung und partielle Differentialgleichungen erster Ordnung. Stuttgart - Leipzig: B. G. Teubner Verlagsges. 1994.

[3] Cesari, L.: Optimization with partial differential equations in Dieudonné-Rashevsky form and conjugate problems. Arch. Rat. Mech. Anal. 33 (1969), 339 - 357.

[4] Clarke, F. H.: Optimization and Non-Smooth Analysis. New York: John Wiley \& Sons, Inc. 1983.

[5]. Dunford, N. and J. T. Schwartz: Linear Operators. Part I: General Theory. New York: Intersci. 1964. 
[6] Kantorowitsch, L. W. and A. P. Akilow: Funktionalanalysis in normierten Räumen. Berlin: Akademie-Verlag 1964.

[7] Kantorovič, L. V.: O peremeščenii mass (in Russian). Dokl. Acad. Nauk SSSR 37 (1942), $227-229$.

[8] Kantorovič, L. V. and M. K. Gawurin: Problems for improving effective working of transport (in Russian). In: The application of Mathematical Methods to Problems of Freight Flow Analysis (eds.: L. V. Kantorovič and M. K. Gawurin). Moscow: Acad. Nauk SSSR 1949, pp.110 - 138.

[9] Klötzler, R.: Pontryagin's maximum principle for multiple integrals. In: System Modelling and Optimization. Lecture Notes in Control and Inform. Sciences: Vol. 180 (ed.: P. Kall). Berlin: Springer-Verlag 1992, pp. 323 - 333.

[10] Klötzler, R. and S. Pickenhain: Pontryagin's Maximum Principle for Multidimensional Control Problems. Intern. Ser. Num. Math. (Birkhäuser Verlag) 111 (1993), 21 - 30.

[11] Klötzler, R.: Flußoptimierung. ZAMM 74 (1994)6, T591 - T593.

[12] Klötzler, R.: Optimal Flows and their Applications. Preprint. Greifswald: Preprint-Reihe Mathematik Nr. 1 (1994), pp. 38 - 41.

[13] Klötzler, R.: Transportfluß-Optimiemıng. Gesellschaft für Mathematik, Ökonomie und Operations Research, Newsletter 2 (1994), 3- 10.

[14] Monge, G.: Sur la théorie des deblais et des remblais. Paris: Mem. Acad. Sci. 1781.

[15] Rolewicz, S.: Funktionalanalysis und Steuerungstheorie. Berlin - Heidelberg - New York: Springer-Verlag 1976.

[16] Yosida, K. and E. Hewitt: Finitely additive measure. Trans. Amer. Soc. 72 (1952), 44 66.

[17] Young, L. C.: Calculus of Variations and Optimal Control Theory. Philadelphia: Saunders Comp. 1969.

Received 28.09.1994 